\title{
New Insights Into the Metabolic Capacity of Gut Microbiota in Coordinating the Efficacy of Mycophenolate Mofetil in Neuromyelitis Optica Spectrum Disorders
}

\section{Gong Li}

South China Agricultural University

Hao Ren

South China Agricultural University

Lijuan Xia

South China Agricultural University

\section{Xiaoyu Ma}

The Third Affiliated Hospital of Sun Yat-sen University

\section{Ran Wei}

South China Agricultural University

\section{Xiran Wang}

South China Agricultural University

Cang Li

South China Agricultural University

\section{Yuge Wang}

The Third Affiliated Hospital of Sun Yat-sen University

\section{Liming He}

South China Agricultural University

Jian Sun ( $\boldsymbol{D}$ jiansun@scau.edu.cn )

South China Agricultural University

Wei Qiu

The Third Affiliated Hospital of Sun Yat-sen University

\section{Research Article}

Keywords: Neuromyelitis optica spectrum disorders, mycophenolate mofetil, gut microbiome $\rrbracket$ therapeutic efficacy, PSP metabolites

Posted Date: February 17th, 2022 
DOI: https://doi.org/10.21203/rs.3.rs-1360750/v1

License: (c) (1) This work is licensed under a Creative Commons Attribution 4.0 International License. Read Full License 


\section{Abstract}

\section{Background}

Mycophenolate mofetil (MMF) has been widely prescribed for the therapy of neuromyelitis optica spectrum disorders (NMOSD) although not all patients display the optimal clinical response to MMF. Our study investigated a potential role of the gut microbiota in the efficacy of MMF in NMOSD patients.

\section{Methods}

A total of 34 NMOSD patients treated with MMF were prospectively enrolled and grouped according to the therapeutic efficacy as effect group (EG) versus non-effect group (NEG). The gut microbiota was analyzed using 16S rRNA sequencing with stool samples and purine metabolites were profiled in serum samples from the same patients. To further decipher the role of the gut microbiota in coordinating the MMF efficacy, metabolic profiling was performed in microbiota-depleted mice.

\section{Results}

The gut microbiota compositions could not distinguish EG and NEG patients although Clostridium and Synergistetes had significantly increased for EG and this corresponded to a significant decline in abundance of the Coprococcus genus. Additionally, purine salvage pathway (PSP) metabolites inosine, hypoxanthine, xanthine, guanine and uric acid in the serum of NMOSD patients were elevated 2 - 7-fold in the NEG group compared to EG $(p<0.05)$. We then used the selected PSP metabolites as indicators of MMF efficacy in a mouse model and found that the MMF response was significantly dampened when the gut microbiota was depleted by vancomycin treatment. The latter microbiomes displayed elevated levels of the PSP metabolites xanthine, inosine and hypoxanthine $(p<0.05)$.

\section{Conclusions}

The activity and metabolic capacities of gut microbiomes are essential for efficient response to MMF in NMOSD patients and implies an active role for the gut microbiota in coordinating host responses to MMF therapy.

\section{Background}

Neuromyelitis optica spectrum disorders (NMOSD) are autoimmune diseases of the central nervous system (CNS) associated with aquaporine (AQP4) channel dysfunctions of astrocytes induced by serum AQP4-IgG autoantibody [1, 2]. Clinically, NMOSD result in a range of syndromes of varied severities including paralysis, blindness and respiratory failure [3]. Immunosuppressive therapies including administration of azathioprine and mycophenolate mofetil (MMF) in addition to the new B cell- and complement-targeted therapies are the current NMOSD treatments [4, 5]. MMF is a prodrug whose active metabolite mycophenolic acid (MPA) inhibits inosine monophosphate dehydrogenase (IMPDH) and suppresses the de novo synthesis of guanine nucleotides in rapidly-proliferating cells such as $T$ and $B$ 
lymphocytes [6, 7]. MMF/MPA treatments significantly and comprehensively lower the levels of baseline purine metabolites in intestinal cell models as well as in patients [8, 9]. However, a subset of NMOSD patients (20 to $50 \%$ ) have no or merely a suboptimal clinical response to MMF treatment [4].

Our laboratory recently demonstrated that the gut microbiomes of healthy individuals can be distinguished from NMOSD patients. In particular, the degree of Streptococcus spp. colonization was significantly and positively correlated with disease severity [10]. Other studies have demonstrated strong associations between several Clostridium spp. and NMOSD development [11, 12]. These studies linked dynamic interactions of the host gut microbiota to NMOSD. However, it is not known whether NMOSD therapies such as MMF administration are also influenced by the gut microbiota and whether the microbiome has a role in the efficacy of these therapies.

In the current study, we performed microbiome analysis and metabolic profiling on samples collected from NMOSD patients and developed a murine model to elucidate whether and how the host gut microbiota participates in regulating MMF efficacy. We found that purine salvage pathway (PSP) metabolites can be used as indicators of the host response to MMF therapy and this can explain how the gut microbiota fine-tunes MMF efficacy (Fig 1).

\section{Materials And Methods}

\section{Reagents and chemicals}

Inosine, hypoxanthine, xanthine, guanine and uric acid were obtained from MedChemExpress (Shanghai, China) and were prepared as $1000 \mathrm{ppm}$ methanol stocks. $\mathrm{NaOH} 10 \mathrm{mM}$ was used as necessary to increase solubility. MMF was purchased from Tokyo Chemical Industry (Shanghai, China) and acetonitrile was purchased from Macklin (Shanghai, China).

\section{Patient enrollment}

The study group included 34 NMOSD patients who had received MMF therapy ( $0.5 \mathrm{~g}$ MMF orally, twice a day for long term therapy) at the Third Affiliated Hospital of Sun Yat-sen University (Guangzhou, China). The primary inclusion criteria were (1) clinical diagnosis of NMOSD and seropositive for AQP4-IgG[13]; (2) patients aged 15-65 and (3) an Kurtzke Expanded Disability Status Scale (EDSS) score $\leq$ 8.0. Patients who met any of the following key criteria were excluded: (1) treatment with antibiotics or probiotics within the last 3 months; (2) emergency treatment such as plasmapheresis or intravenous methylprednisolone for acute recurrence within the last 1 month; (3) Concurrent disease with diabetes mellitus, systemic autoimmune diseases or other neurological diseases.

Stool and serum samples were taken from the patient for $0.5,1$ and $2 \mathrm{~h}$ after receiving MMF in the early morning. Clinical data that was collected included follow-up time, relapses and EDSS score. The 34 participants were divided into two groups; patients that had decreased CNS symptoms and decreased 
EDSS scores were assigned to the effect group (EG) and patients whose symptoms were not altered were treated as the non-effect group (NEG) [14].

This study was approved by the ethics Committee of the Third Affiliated Hospital of Sun Yat-sen University ([2020]02-016-01) and all patients signed informed consent.

\section{Microbiome 16S rRNA gene sequencing, alignment and analysis}

Microbial DNA was extracted from fecal samples of NMOSD patients using the E.Z.N.A. Stool DNA Kit (Omega Bio-Tek, Norcross, GA, USA) according to the manufacturer's protocol. Extracted DNA was qualitatively and quantitatively analyzed by gel electrophoresis and ultraviolet spectroscopy before sequencing. Thereafter, bacterial $16 \mathrm{~S}$ rDNA V3-V4 regions were amplified using primers $341 \mathrm{~F}$ and $806 \mathrm{R}$ (Illumina, San Diego, CA, USA). The resulting amplicons were sequenced using the Illumina NovaSeq 6000 platform (Guangdong Longsee Biomedical, Foshan, China).

\section{HPLC-MS/MS analysis}

Stool sample processing and HPLC-UV-MS/MS analysis were performed as previously described with minor modifications [15]. In brief, chromatographic separations were conducted using a Shimadzu LC30AD series HPLC system (Shimadzu, Kyoto, Japan) with an Frulic N column (AZYP, LLC, Arlington, USA). Analytes were eluted at a flow rate of $0.4 \mathrm{~mL} / \mathrm{min}$ using a gradient of phase $\mathrm{A}(0.2 \%$ formic acid) and phase B (0.2\% formic acid in acetonitrile) as follows: 0-0.5 min, $80 \%$ B; $0.5-4.5 \mathrm{~min}, 50 \% \mathrm{~B} ; 4.5-8 \mathrm{~min}$, $80 \% \mathrm{~B}$. The eluates were monitored by UV at $254 \mathrm{~nm}$ and MS detection in parallel. The injection volume as $5 \mu \mathrm{L}$, and the column temperature was maintained at $23^{\circ} \mathrm{C}$. MS detection was performed using an $A B$ Sciex Triple Quad 5500 (AB Sciex, Toronto, Canada). The MS source parameters were set as follows: drying gas temperature, $300^{\circ} \mathrm{C}$; gas flow rate, $10 \mathrm{~L} / \mathrm{min}$; sheath gas temperature, $350^{\circ} \mathrm{C}$; sheath gas flow rate, $12 \mathrm{~L} / \mathrm{min}$; nebulizer, 45 psig; capillary voltage, $3500 \mathrm{~V}$; and nozzle voltage, $500 \mathrm{~V}$. Nitrogen was used as the drying and collision gas. Detection was conducted in positive mode using multiple reaction monitoring (MRM).

\section{Animal trials}

Female C57BL/ 6 mice aged 6 weeks were used in all experiments. Animals were maintained in Laboratory animal center of South China Agricultural University (Guangzhou, China). Mice were housed in sterilized, filtertop cages under specific pathogen-free conditions and fed control diets for a minimum of 1 week before commencing experiments. Vancomycin was administered in drinking water for 14 days in advance to deplete part of gut microbiome and then fed with $0.563 \%(\mathrm{w} / \mathrm{w}) \mathrm{MMF}$ for 7 consecutive days and designated as Van-MMF combined intervention group (VM). The MMF group were fed MMF for 7 days without vancomycin treatment and controls received normal feed and drinking water (Fig. 3A). MMF was supplemented at $0.563 \%(\mathrm{w} / \mathrm{w})$ into the diets for experimental groups. All of the animal experiments adhered to the institution's guidelines for animal use and the Guide for the Care and use of Laboratory Animals at laboratory animal center of South China Agricultural University (Guangzhou, China). Whole 
blood was collected from anesthetized animals by cardiac puncture. Serum were isolated by centrifugation at $3000 \mathrm{rpm}$ for $10 \mathrm{~min}$ and stored at $-80^{\circ} \mathrm{C}$.

\section{Metabolome profiling}

Metabolomes of mouse serum were profiled using a GC-MS-based untargeted metabolomic procedure[16]. The samples were added into an Eppendorf tube with $200 \mu \mathrm{L}$ extractant solution (acetonitrile: methanol $=1: 1$, including internal standard of isotope-labeled winged peptide) and vortexed for $30 \mathrm{~s}$ and then incubated at $-40^{\circ} \mathrm{C}$ for $1 \mathrm{~h}$. The samples were centrifuged at $12000 \mathrm{rpm}$ for $15 \mathrm{~min}$ at $4^{\circ} \mathrm{C}$ and the resulting supernatant was transferred to a clean glass vial for analysis as described previously [17]. The quality control (QC) sample was the combination of the supernatants from all of the samples.

\section{LC-MS/MS analysis}

LC-MS analyses were performed using a UHPLC system (Vanquish, Thermo Fisher Scientific) with a UPLC BEH Amide column $(2.1 \mathrm{~mm} \times 100 \mathrm{~mm}, 1.7 \mu \mathrm{m})$ coupled to a Q Exactive HAF Mass Spectrometer (Orbitrap MS, Thermo Fisher, Pittsburg, PA, USA). Mobile phase A was consisted of $25 \mathrm{mmol} / \mathrm{L}$ ammonium acetate and 25 ammonia hydroxide in water $(\mathrm{pH}=9.75)$. Mobile phase $\mathrm{B}$ was acetonitrile. The injection volume was $3 \mu \mathrm{L}$. The QE HFX mass spectrometer was used for its ability to acquire MS/MS spectra on information-dependent acquisition (IDA) mode in the control of the Xcalibur 4.0.27 acquisition software (Thermo Fisher). In this mode, the acquisition software continuously evaluates the full scan MS spectrum. The electrospray ionization (ESI) source conditions were set as follows: sheath gas flow rate $30 \mathrm{arb}$, aux gas flow rate 25 arb, capillary temperature $350^{\circ} \mathrm{C}$, full MS resolution as 60,000 , MS/MS resolution as 7,500, collision energy as $10 / 30 / 60$ in NCE mode and spray voltage as $4.0 \mathrm{kV}$ (positive) or $3.6 \mathrm{kV}$ (negative).

\section{Data processing and statistics}

Raw data were converted into the mzXML format using ProteoWizard and processed for peak detection, extraction, alignment and integration with an in-house program that was developed using $\mathrm{R}$ and based on XCMS (Smith et al., 2006). An in-house MS2 database (Biotree DB, Shanghai, China) was applied for metabolite annotations with a mass tolerance of $10 \mathrm{ppm}$. The cutoff for annotation was set at 0.3 and 6068 peaks were detected and 279 metabolites remained after noise reduction relative to the standard deviation in serum samples and the internal standard was used for data normalization. The final dataset contained the peak number, sample name and normalized peak area and was imported into the SIMCA 15.0.2 software package (Sartorius Stedim Data Analytics (Umea, Sweden). Data were scaled and logtransformed and further analyzed as previously described [17].

\section{Statistical Analyses}

The data were expressed as the mean \pm standard deviation (SD). A nonparametric T-test was performed for comparison of the two groups using Prism v8.0 software (GraphPad, San Diego, CA, USA). A $p$ value 
of 0.05 was considered to be statistically significant.

\section{Results}

\section{The gut microbiota of NMOSD patients were not compositionally distinguished by their responses to MMF therapy}

Previous studies have indicated that the gut microbiome participates in the development and pathogenesis of NMOSD[10-12]. Our group of NMOSD patients were analyzed according to their clinical responses to MMF therapies and grouped according to responders (EG) and non-responders (NEG). The gut microbiota were profiled for each patient using 16S rRNA sequencing (Table 1). The microbial compositions of each group were compared using the 20 most-abundant operational taxonomic units (OTU) at the genus level. Overall, Bacteroides dominated in both EG and NEG patients while the other dominate genera including Faecalibacterium, Roseburia and Megamonas, rarely exceeded $30 \%$ of all sequences (Fig 2A). Taken together, the gut microbiota of EG and NEG patients were not obviously different. Furthermore, the microbial richness (OTUs and Chao1 indices) were similar as was microbial diversity (Shannon and Simpson indices) (Fig. 2B -E). $\beta$ diversity was assessed using principal-coordinate analysis (PCA) based on weighted UniFrac metrics and the results again indicated no compositional alterations between the microbiota of the patients in the two groups (Fig 2E).

Since no structural shifts were observed among the patients in their response to MMF, we extended our taxonomic comparisons. Interestingly, LEfSe analysis indicated that the abundance of Clostridium and Synergistetes had significantly increased for EG and corresponded to a significant decline in abundance of the Coprococcus genus in NEG (Fig 2G). These results indicated that overall, the gut microbiota stayed compositionally stable in the presence and absence of MMF therapy while the abundance of 3 bacterial genera were significantly altered. Therefore, we hypothesized that the therapeutic efficacy of MMF may be attributed to the interplay of the bacterial activity of gut microbiota and its interaction with the polymorphism of the host and more specifically, with the MMF target gene product inosine-5'monophosphate dehydrogenase (IMPDH).

\section{Purine salvage pathway metabolites are maintained at high levels in NEG patients}

To verify our hypothesis, we sought to determine whether MMF interacts with bacterial activity of the gut microbiota in a murine model. However, appropriate indicators to reflect MMF efficacy in animal models are lacking. We therefore examined purine metabolites whose biosynthesis is targeted by MMF in the serum of recruited patients (Fig 3A and 3B). Interestingly, we found distinct differences in the abundance of the purine salvage pathway (PSP) metabolites inosine, hypoxanthine, xanthine, guanine and uric acid (UA) for the patients that responded to MMF therapy (EG) and non-responders (NEG). These 5 PSP metabolites were all elevated in the serum of NEG patients. In particular, inosine, hypoxanthine, xanthine and guanine were significantly $(p<0.05)$ decreased in EG patients $1 \mathrm{~h}$ after receiving the MMF. At $2 \mathrm{~h}$ following MMF intervention, inosine levels reached the level of significance $(p<0.05)$ and were also lower in EG patients (Fig. 3C-G). The observations indicated a negative correlation between the MMF 
therapeutic response and PSP metabolite levels in serum of patients. These data also suggested that PSP metabolites can be used to assess MMF efficacy.

\section{Loss of bacterial activity by microbiota depletion drastically altered the serum metabolome in MMF- treated mice.}

We further investigated MMF responses using mice that had undergone microbiota depletion by vancomycin (VM) administration (Fig. 4A). We applied PCA to determine whether we could identify metabolic heterogeneity in serum samples between untreated controls and MMF and VM treatment groups. Interestingly, the PCA results indicated that the 3 groups clustered separately within the $95 \%$ confidence intervals using Hotelling's T-squared ellipse. Thus, the metabolomes of these mice were significantly altered after MMF administration and depletion of gut microbiota led to a pronounced metabolic shift among the animals that received MMF (Fig 4B).

We also identified 8 metabolic pathways that were significantly altered by MMF treatment as assessed by their (impact) scores. These pathways included phenylalanine, tyrosine and tryptophan biosynthesis $(0.5)$, histidine metabolism $(0.145)$, tyrosine metabolism $(0.140)$, pyrimidine metabolism $(0.133)$, pentose and glucuronate interconversions (0.125), galactose metabolism (0.064), purine metabolism (0.051) and steroid hormone biosynthesis (0.016) (Fig. 4C). Additionally, 56 endogenous metabolites were recognized as different metabolites in the serum samples and showed significant fluctuations (Fig 4E). Moreover, 9 metabolic pathways were highly responsive to the depletion of the gut microbiota and could be distinguished from animals receiving MMF. These pathways included vitamin B6 metabolism (0.490), histidine metabolism (0.145), galactose metabolism (0.064), citrate cycle (TCA cycle) (0.053), purine metabolism (0.050), pyrimidine metabolism (0.048), tryptophan metabolism $(0.018)$, steroid hormone biosynthesis (0.016) and alanine, aspartate and glutamate metabolism (0.003) (Fig 4D). Interestingly, 30 of the differentially-regulated metabolites induced by MMF were reversed by the depletion of gut microbiota and all involved the PSP metabolites xanthine, inosine and hypoxanthine as well as the active form of MMF (mycophenolic acid) (Fig 4E).

\section{Gut microbiome dysbiosis possibly dampens the efficacy of MMF therapy}

Given the efficacy-indicating function of the PSP metabolites xanthine, inosine and hypoxanthine in MMF therapy, they were filtered out to decipher the role of gut microbiota in interaction with therapeutic response of patients to MMF. In particular, vancomycin in combination with MMF significantly decreased MPA levels but increased the levels of selected PSP metabolites with the exception of xanthosine in mice compared with mice that only received MMF (Fig. 5A - E). These results indicated that the loss of bacterial activity by gut microbiota dysbiosis dampened MMF efficacy by reducing the bioavailability of MPA.

\section{Discussion}


NMOSD pathogenesis has been linked to the activity of microbiota [18] but whether the microbiome is also involved in the therapeutic response to the drugs against NMOSD has not been previously investigated. In this study, we profiled the microbiomes of NMOSD patients that were grouped according to their therapeutic responses to MMF therapy. Surprisingly, we found that the microbiota compositions of the MMF non-responders did not significantly differ from the responders. This implied a minor impact of the established host microbiome on the therapeutic response to MMF. In contrast, the abundance of Coprococcus was markedly elevated in EG patients and sharply reduced in the non-responder groups [19]. The genus Coprococcus includes species that are strong butyrate producers [20, 21] so that one possible explanation for our results is that they alleviate the syndromes of NMOSD by butyrate-dependent anti-inflammatory effects as well as having a negative effect on IL-17A expression[22, 23]. Hence, the existing Coprococcus species might be beneficial microbes to patients with NMOSD and ameliorate its symptoms by curtailing the levels of pro-inflammatory cytokines.

A key factor for the poor response to MMF therapy in NMOSD patients has been linked to host genetic polymorphisms. For instance, single nucleotide polymorphisms (SNPs) in the genes encoding the MPA metabolizing enzyme UDP-glucuronosyltransferase 1-9 (UGT1A9) or the molecular targets (IMPDH1 and IMPDH2) are linked to a weakened drug responsiveness of individual patients [20,31]. In addition to SNP differences, the metabolic capacity of gut microbiota also actively participates in the utilization and metabolism of MMF e.g. through $\beta$-glucuronidase (GUS) enzymes expressed by gut commensals [24]. Our results indicated that the MMF therapeutic response was not strongly associated with the gut microbiota composition. This might indicate that the microbiota may contribute to MMF efficacy by their bacterial activity in metabolizing and recycling the active compounds. Consistent with this hypothesis we found that the levels of PSP metabolites could distinguish MMF responders from non-responders. MMF was originally designed to inhibit GMP and GTP synthesis. However, GTP synthesis is also coupled to purine de novo synthesis via PRPP availability that is regulated by the rapamycin complex 1 (mTORC1) [25]. Thus, other purines and in particular the PSP metabolites should be suppressed by the presence of MMF. In support of this, previous studies have demonstrated that levels of PSP metabolites levels in serum or plasma are elevated in patients with CNS disease [26,27]. Similarly, UA levels were mildly elevated in NMOSD patients during relapse compared with patients with a good prognosis [28].These findings coupled with our results indicated that selected PSP metabolites might be used to verify the therapeutic efficacy of MMF in NMOSD patients.

We used the levels of these selected PSPs as indicators to explore whether the bacterial activity of gut microbiota contribute to the MMF efficacy in animal models of mice that either received MMF alone or with microbiota-eradicating vancomycin. The PSP metabolites were maintained at significantly higher levels in the vancomycin-treated mice after administration of MMF. This suggested that the MMF might fail to regulate purine metabolism in absence of gut microbiota. We constructed a scenario where the orally-consumed MMF is converted to active MPA in the stomach and circulated to in situ sites to exert inhibitory effects on NMOSD. These are then processed by hepatic UGT enzymes that create inactive glucuronidated (MPAG) and acetylated (ACMPAG) MPA in the liver and a portion are transported by ABCC2 into the GI tract. The bacterial GUS enzymes remove the GA moiety to produce free MPA which 
undergoes enterohepatic recirculation (see Fig 5E) [24]. Our results demonstrated that depletion of GUSexpressing bacteria with vancomycin dampened the efficacy of MMF, possibly due to impaired MPA recruitment from haematogenetic circulation, leading to reduced MPA bioavailability. Hence, although vancomycin was reported to relieve MMF-induced gastrointestinal toxicity [24], this type of treatment could actually weaken MMF efficacy.

\section{Conclusions}

In this study, we found that an intact gut microbiota was linked to MMF efficacy. In particular, GUSproducing bacteria among the gut microbiota may potentiate MMF therapy by enhancing the bioactivity of active MPA via hepatoenteric circulation. Collectively, this study sheds light on the active role of the gut microbiota in coordinating the host response to MMF therapy and the potential poor response to MMF therapy in the patients undergoing gut microbiota dysbiosis.

\section{Declarations}

\section{Acknowledgements}

We thank Hangzhou Zhongmei Huadong Pharmaceutical for their help with grant support.

\section{Authors' contributions:}

GL, LJX, XYM, RW, CL, LMH contributed to the acquisition of data, GL, HR, XRW, YGW contributed to the analysis of data. GL, HR, and WQ contributed to the drafting of the work. JS and WQ contributed to the conception and design drafting of the work. All authors read and approved the final manuscript.

\section{Funding}

This work was supported by grants from the National Natural Science Foundation of China (81771300, 81971140) $₫$ Natural Science Foundation of Guangdong Province (2020A1515010053) and the Guangdong Major Project of Basic and Applied Basic Research (grant 2020B0301030007) .

\section{Availability of data and materials}

No supporting data besides presented in the manuscript.

\section{Ethics approval and consent to participate}

The study was approved by the ethics Committee of the Third Affiliated Hospital of Sun Yat-sen University ([2020]02-016-01), and all patients signed informed consent. Animal experimentation was approved by the by the ethics Committee of the laboratory animal center of South China Agricultural University (Guangzhou, China), approval number 2020B041.

\section{Consent for publication}


Not applicable.

\section{Competing interests}

The authors declare that they have no competing interests.

\section{References}

1. Cruz, R., et al., Neuromyelitis Optica Spectrum Disorders (NMOSD) and Connective Tissue Disease (CTD): an Update for the Rheumatologist. Current rheumatology reports, 2021. 23(6): p. 33.

2. Lucchinetti, C., et al., The pathology of an autoimmune astrocytopathy: lessons learned from neuromyelitis optica. Brain pathology (Zurich, Switzerland), 2014. 24(1): p. 83-97.

3. Sellner, J., et al., EFNS guidelines on diagnosis and management of neuromyelitis optica. European journal of neurology, 2010. 17(8): p. 1019-32.

4. Huang, Q., et al., Low-Dose Mycophenolate Mofetil for Treatment of Neuromyelitis Optica Spectrum Disorders: A Prospective Multicenter Study in South China. Frontiers in immunology, 2018. 9: p. 2066.

5. Wingerchuk, D. and B. Weinshenker, Neuromyelitis optica. Current treatment options in neurology, 2008. 10(1): p. 55-66.

6. Allison, A., Mechanisms of action of mycophenolate mofetil. Lupus, 2005: p. s2-8.

7. Allison, A.C. and E.M. Eugui, The design and development of an immunosuppressive drug, mycophenolate mofetil. Springer Semin Immunopathol, 1993. 14(4): p. 353-80.

8. Moreno Planas, J., et al., Mycophenolate mofetil can be used as monotherapy late after liver transplantation. American journal of transplantation : official journal of the American Society of Transplantation and the American Society of Transplant Surgeons, 2004. 4(10): p. 1650-5.

9. Heischmann, S., et al., The Immunosuppressant Mycophenolic Acid Alters Nucleotide and Lipid Metabolism in an Intestinal Cell Model. Sci Rep, 2017. 7: p. 45088.

10. Gong, J., et al., Lack of short-chain fatty acids and overgrowth of opportunistic pathogens define dysbiosis of neuromyelitis optica spectrum disorders: A Chinese pilot study. Multiple sclerosis (Houndmills, Basingstoke, England), 2019. 25(9): p. 1316-1325.

11. Pandit, L., et al., Clostridium bolteae is elevated in neuromyelitis optica spectrum disorder in India and shares sequence similarity with $A Q P 4$. Neurology $(\mathrm{R})$ neuroimmunology \& neuroinflammation, 2021. 8(1).

12. Cree, B., et al., Gut microbiome analysis in neuromyelitis optica reveals overabundance of Clostridium perfringens. Annals of neurology, 2016. 80(3): p. 443-7.

13. Wingerchuk, D., et al., International consensus diagnostic criteria for neuromyelitis optica spectrum disorders. Neurology, 2015. 85(2): p. 177-89.

14. Liu, L., et al., Effect of inosine monophosphate dehydrogenase-1 gene polymorphisms on mycophenolate mofetil effectiveness in neuromyelitis optica spectrum disorder patients. Multiple sclerosis and related disorders, 2021. 49: p. 102779. 
15. Lu, J.J., et al., Ultra-high performance liquid chromatography with ultraviolet and tandem mass spectrometry for simultaneous determination of metabolites in purine pathway of rat plasma. $\mathrm{J}$ Chromatogr B Analyt Technol Biomed Life Sci, 2016. 1036-1037: p. 84-92.

16. Dunn, W.B., et al., Procedures for large-scale metabolic profiling of serum and plasma using gas chromatography and liquid chromatography coupled to mass spectrometry. Nature Protocols, 2011. 6(7): p. 1060-1083.

17. Zhou, L., D. Wang, and H. Zhou, Metabolic profiling of two medicinal Piper species. South African Journal of Botany, 2021. 139: p. 281-289.

18. Cui, C., Y. Ruan, and W. Qiu, Potential role of the gut microbiota in neuromyelitis optica spectrum disorder: Implication for intervention. J Clin Neurosci, 2020. 82(Pt B): p. 193-199.

19. Shi, Z., et al., Dysbiosis of gut microbiota in patients with neuromyelitis optica spectrum disorders: A cross sectional study. Journal of neuroimmunology, 2020. 339: p. 577126.

20. Liu, C., et al., Study on fecal fermentation characteristics of aloe polysaccharides in vitro and their predictive modeling. Carbohydr Polym, 2021. 256: p. 117571.

21. Maier, L., et al., Extensive impact of non-antibiotic drugs on human gut bacteria. Nature, 2018. 555(7698): p. 623-628.

22. Huang, S., et al., The imbalance of gut microbiota and its correlation with plasma inflammatory cytokines in pemphigus vulgaris patients. Scandinavian journal of immunology, 2019. 90(3): p. e12799.

23. Yeh, N., et al., Gut Microbiome in Psoriasis is Perturbed Differently During Secukinumab and Ustekinumab Therapy and Associated with Response to Treatment. Clinical drug investigation, 2019. 39(12): p. 1195-1203.

24. Taylor, M., et al., Vancomycin relieves mycophenolate mofetil-induced gastrointestinal toxicity by eliminating gut bacterial $\beta$-glucuronidase activity. Science advances, 2019. 5(8): p. eaax2358.

25. Emmanuel, N., et al., Purine Nucleotide Availability Regulates mTORC1 Activity through the Rheb GTPase. Cell Rep, 2017. 19(13): p. 2665-2680.

26. Lazzarino, G., et al., Serum Compounds of Energy Metabolism Impairment Are Related to Disability, Disease Course and Neuroimaging in Multiple Sclerosis. Molecular neurobiology, 2017. 54(9): p. 7520-7533.

27. Kuračka, L., et al., Multiple sclerosis: evaluation of purine nucleotide metabolism in central nervous system in association with serum levels of selected fat-soluble antioxidants. Multiple sclerosis international, 2014. 2014: p. 759808.

28. Shu, Y., et al., Elevated cerebrospinal fluid uric acid during relapse of neuromyelitis optica spectrum disorders. Brain and behavior, 2017. 7(1): p. e00584.

\section{Tables}

Table 1. Clinical parameters in NMOSD patients according to response to MMF. 


\begin{tabular}{|llll|}
\hline Variables & EG $(\mathbf{n}=23)$ & NEG $(\mathbf{n}=11)$ & $p$ value \\
\hline Age (years) & $39.95 \pm 12.20$ & $47.45 \pm 11.76$ & 0.106 \\
\hline Gender (male: female) & $5: 18$ & $1: 10$ & 0.638 \\
\hline Follow-up time (months, median (range)) & $21(6-48)$ & $37(10-59)$ & 0.060 \\
\hline Relapse time (median (range)) & $0(0-0)$ & $2(1-4)$ & 0.000 \\
\hline AQP4-ab positive & 23 & 11 & 1 \\
\hline EDSS before MMF (median(range)) & $4(2-6)$ & $3.5(1.5-5)$ & 0.228 \\
\hline EDSS after MMF (median(range)) & $3(0-4.5)$ & $4(2.5-7)$ & 0.005 \\
\hline
\end{tabular}

Figures 
Poor therapeutic efficacy



1. 16S rRNA sequencing for fecal samples

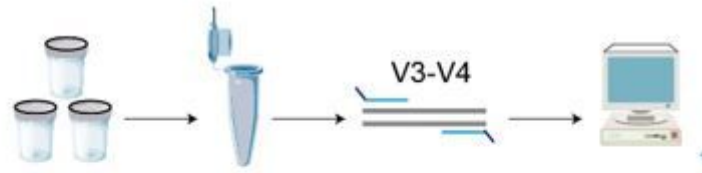

Stool collection from NMOSD patients

2. HPLC-MS/MS analysis for serum PSP metabolites



Serum collection from NMOSD patients

3. PSP metabolites analysis in microbiota elimination model

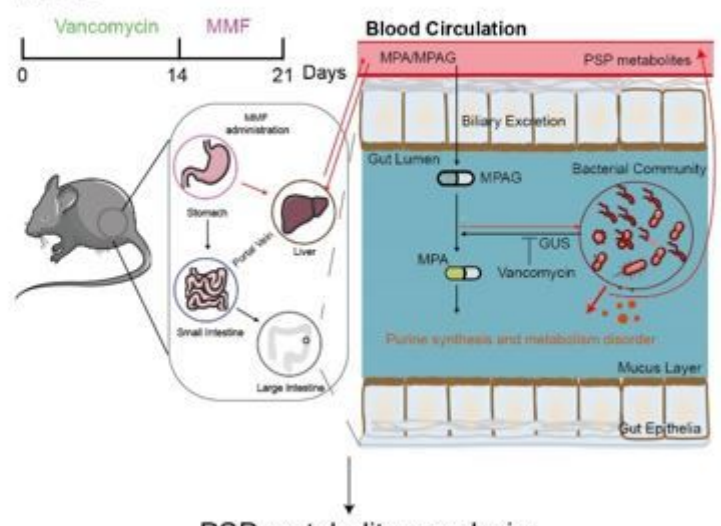

PSP metabolites analysis

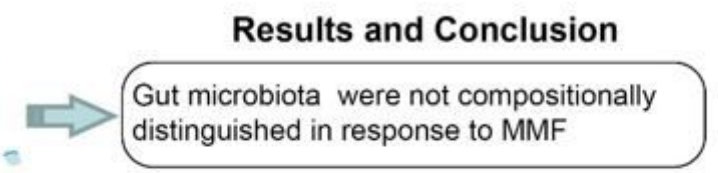

Exploring indicator for Therapeutic efficacy therapy in mice model

Applying PSP

metabolites as therapeutic efficacy indicator

$\downarrow$



GUS-producing bacteria may potentiate the MMF therapy

\section{Figure 1}

Schematic representation of experimental procedures used in this study. 

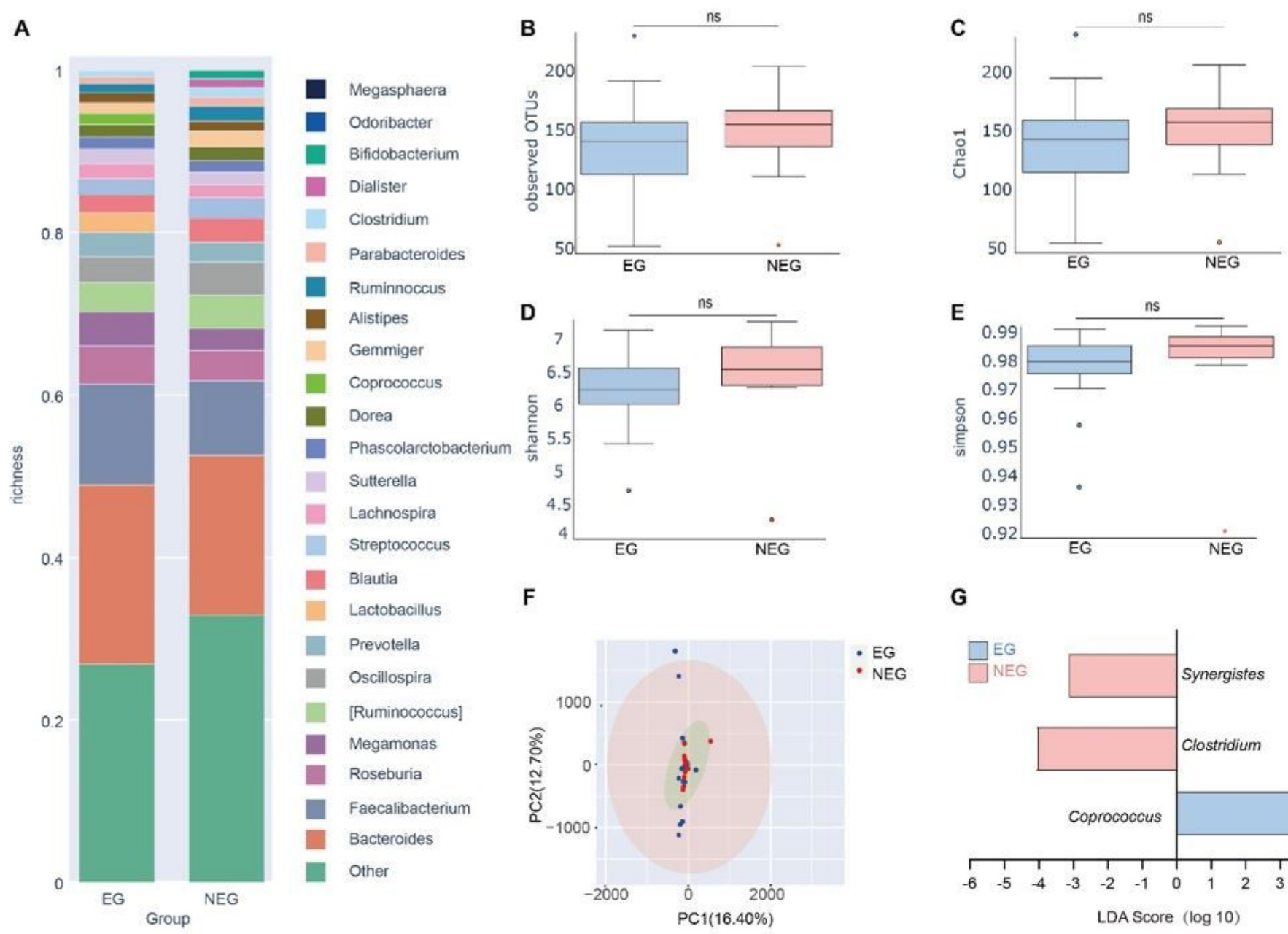

G

Lactobacillus

F

Prevotella

Oscillospira

[Ruminococcus]

Megamonas

Roseburia

Faecalibacterium

Bacteroides

Other


Figure 2

The gut microbiota of NMOSD patients were not compositionally distinguished by their responses to MMF therapy. (A) Composition of the gut microbiome at the genus levels in fecal samples in EG and NEG samples. (B) Observed OTUs. (C) Chao1 index. (D) Shannon index. (E) Simpson index. (F) Principal component analysis. (G) LEfSe (Linear discriminant analysis effect size) analysis showing taxa associated with therapeutic response of MMF. $N=23$ for effect group (EG) and $n=11$ for non-effect group (NEG). ${ }^{*} p<0.05$. 
A

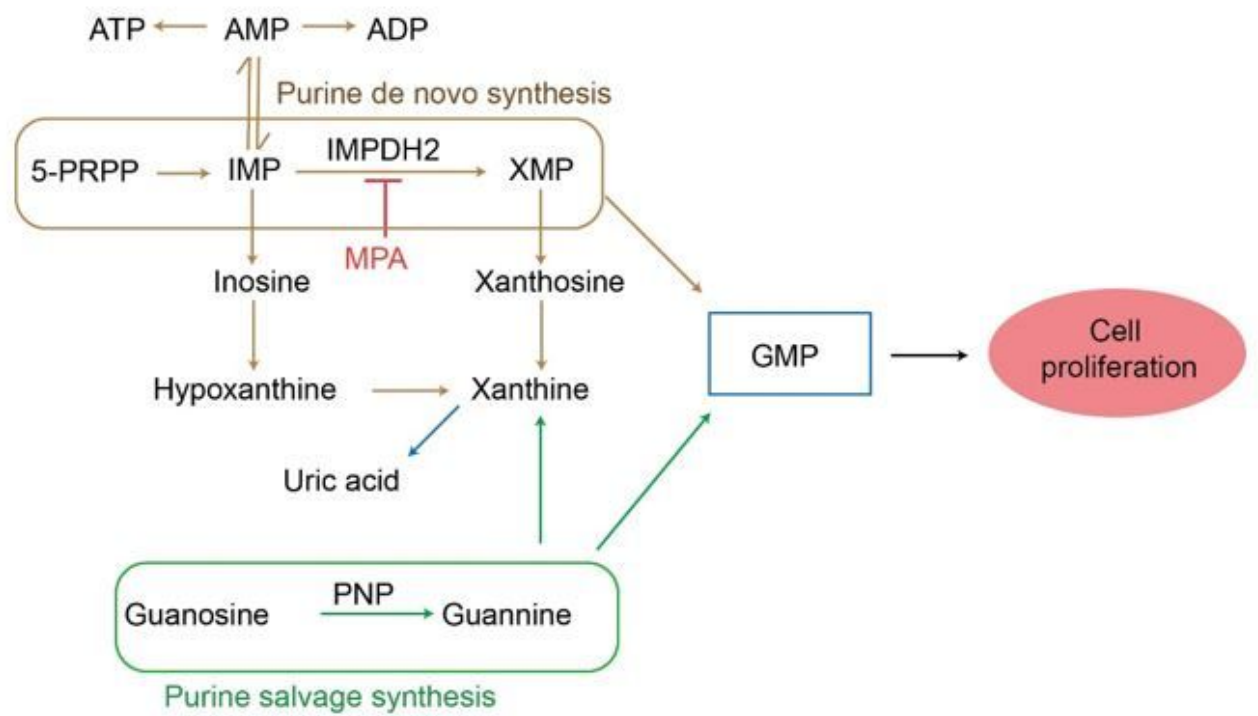

B
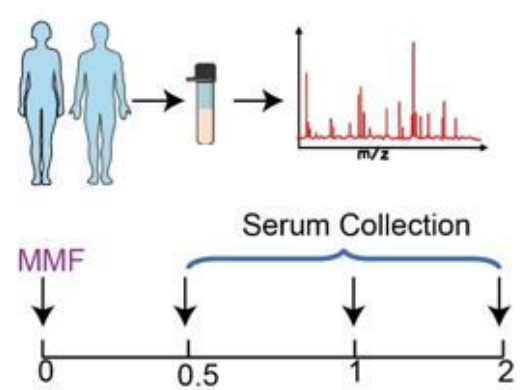

E

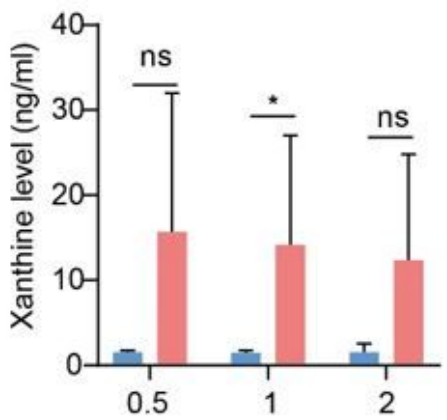

C



F

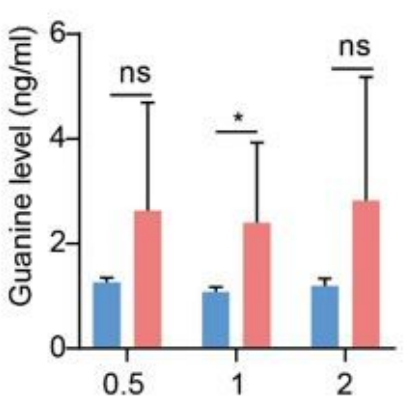

EG

NEG
D

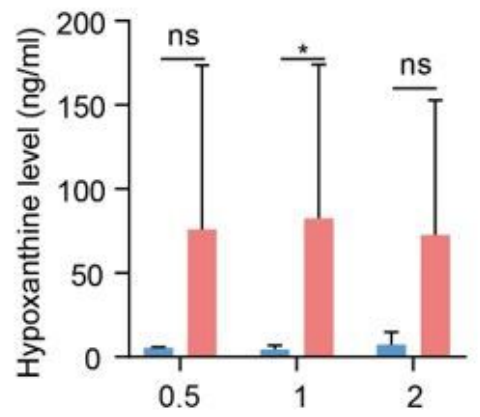

G

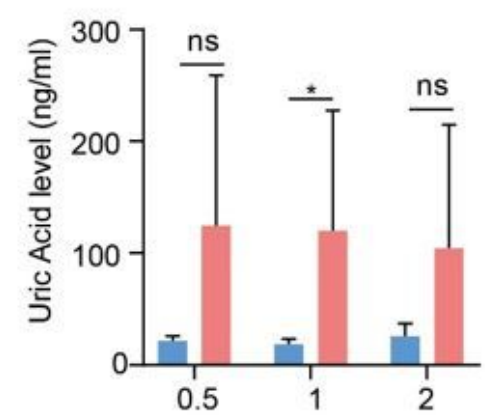

\section{Figure 3}

Purine salvage pathway metabolites are maintained at high levels in NEG patients. (A) Schematic of the de novo and salvage pathways of purine synthesis. (B) Serum collection point for the experiments. Quantification of the levels of (C) Inosine (D) Hypoxanthine (E) Xanthine (F) Guanine and (G) Uric acid. * $p$ $<0.05$ 
A

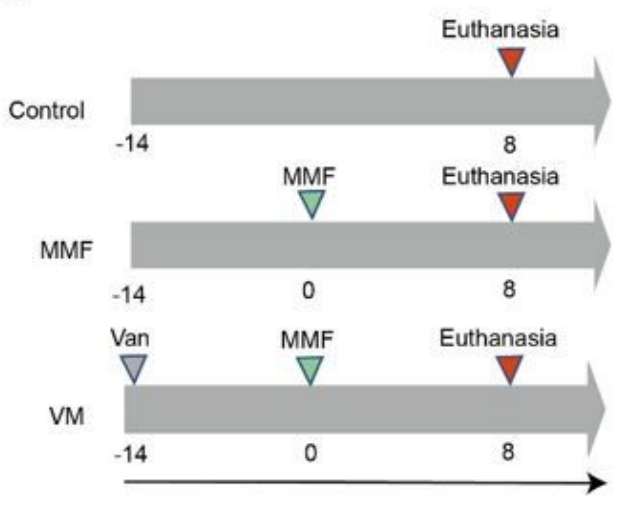

C

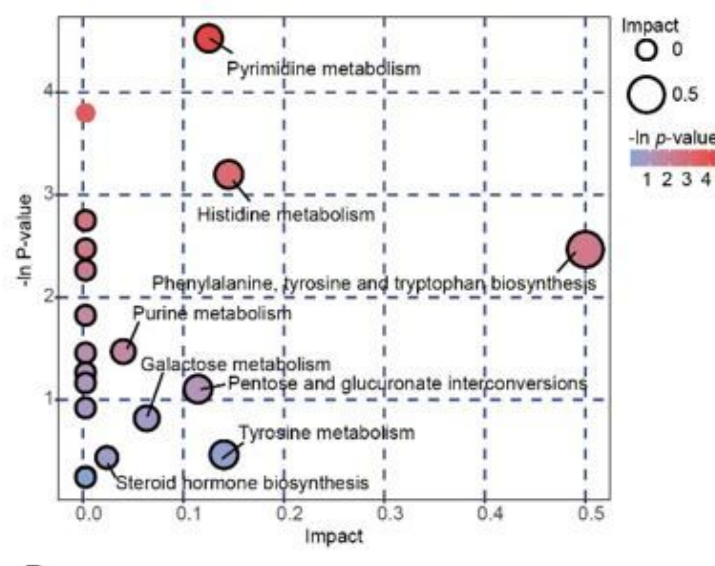

D

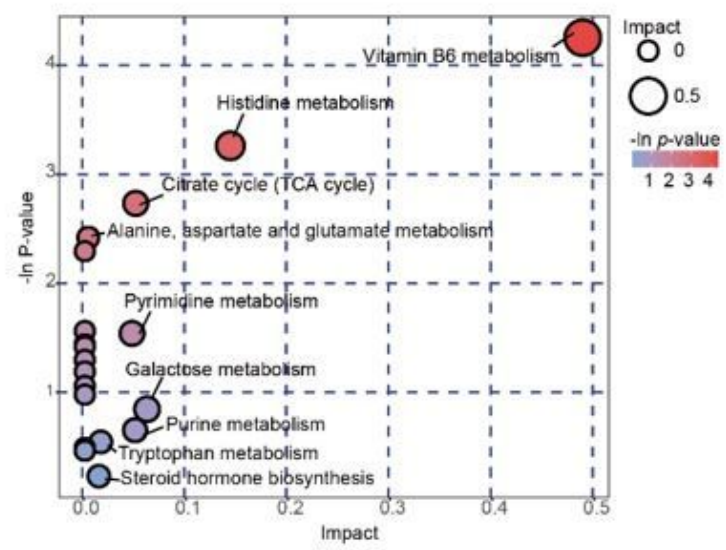

B

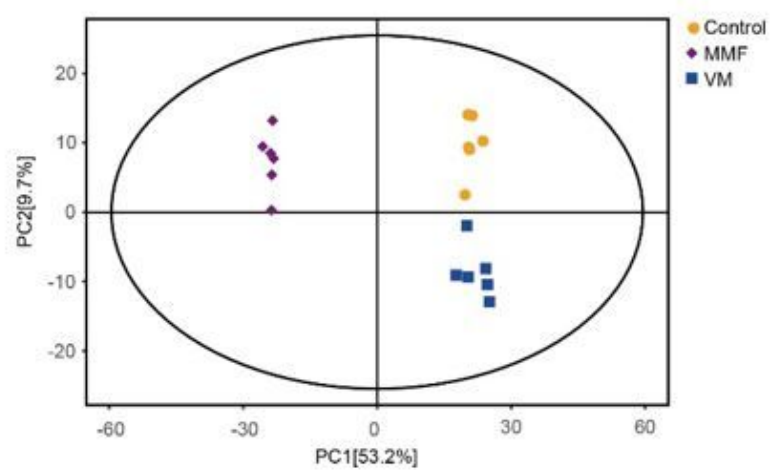

E

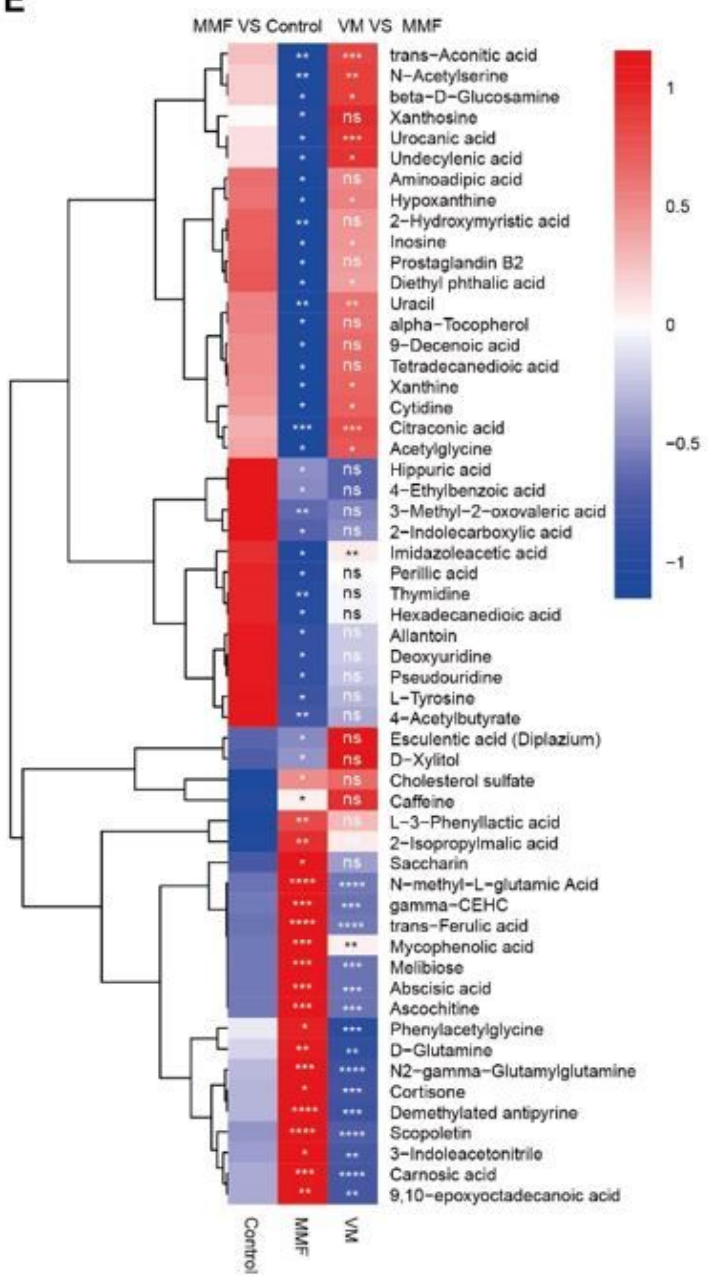

\section{Figure 4}

Loss of bacterial activity by microbiota depletion drastically altered the serum metabolome in MMFtreated mice. (A) Setup of the experimental procedures. (B) Principal component analysis (PCA) of Control, MMF and VM groups in colon samples. (C) Differential metabolic pathways of MMF group compared to Control mice. (D) Differential metabolic pathways of VM group to MMF-induced colitis mice. 
(E) Heatmap of differential metabolites between Control, MMF and VM groups in serum. $(n=5 \sim 6$ mice/group). VM, vancomycin.

A
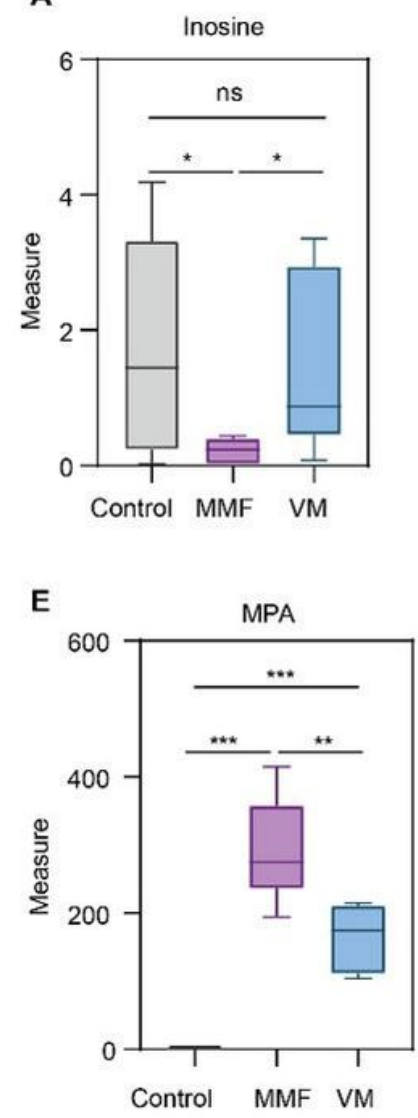

B



C

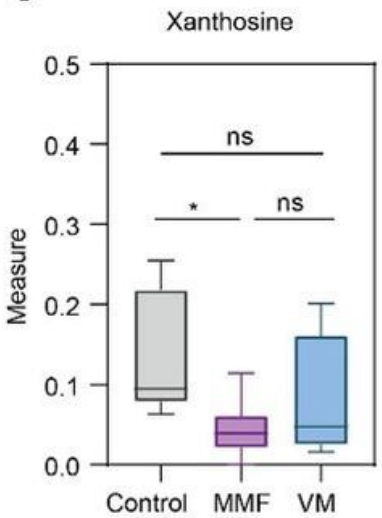

D

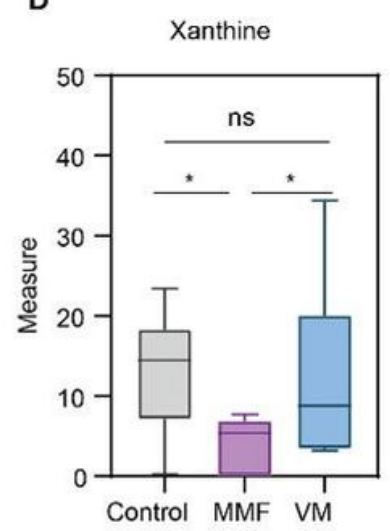

F

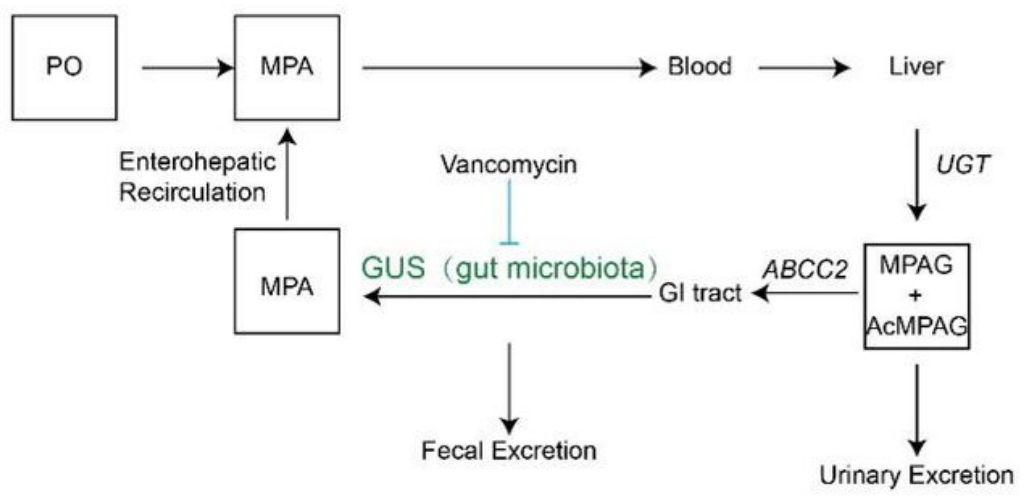

\section{Figure 5}

Gut microbiome dysbiosis possibly dampens the efficacy of MMF therapy. (A) Inosine levels in serum of the indicated experimental groups ( $n=5-6$ mice/group). Quantification of (B) Hypoxanthine (C) Xanthosine (D) Xanthine and (E) MPA. (F) MMF metabolism. ${ }^{*} p<.05$. 